\title{
Relationships between Certain Pyrimidine and Arginine Mutants of Neurospora, as Revealed by their Response to Carbon Dioxide
}

\author{
BY H. P. CHARLES \\ Department of Microbiology, The University, Reading, England
}

(Received 12 August 1963)

\begin{abstract}
SUMMARY
A pyrimidine-requiring mutant of Neurospora was found to grow on minimal medium when the proportion of $\mathrm{CO}_{2}$ in the gas-phase (air) was increased to $30 \%(\mathrm{v} / \mathrm{v})$; arginine prevented growth in $30 \% \mathrm{CO}_{2}$, but did not prevent growth when pyrimidines were present. An arginine-requiring mutant also grew on minimal medium in the presence of $30 \% \mathrm{CO}_{2}$. The response of this mutant to $\mathrm{CO}_{2}$ was annulled by pyrimidines. It is proposed that these mutants have defects in carbamoyl phosphate synthesis. Wild-type Neurospora may have two pathways for making carbamoyl phosphate from $\mathrm{CO}_{2}$, one pathway subject to feed-back inhibition or repression by arginine, the other pathway subject to feed-back inhibition or repression by pyrimidines. The pyrimidine mutant may lack an enzyme which produces carbamoyl phosphate for pyrimidine synthesis, and the arginine mutant may lack an enzyme which produces carbamoyl phosphate for arginine synthesis. Thus each mutant may have only one effective mechanism for carbamoyl phosphate synthesis. It is suggested that $30 \% \mathrm{CO}_{2}$ causes increased synthesis of carbamoyl phosphate by the remaining pathway in each mutant, thereby providing sufficient carbamoyl phosphate to overcome the nutritional deficiency. The inhibition of the pyrimidine mutant by arginine, and the inhibition of the arginine mutant by pyrimidines, are explained as feed-back inhibition or repression of the remaining pathway for carbamoyl phosphate synthesis.
\end{abstract}

\section{INTRODUCTION}

This paper describes interrelations between certain uridine-requiring and arginine-requiring mutants of Neurospora crassa. The interrelations are expressed in two ways. First, both mutants grow vigorously on minimal medium when the proportion of carbon dioxide $\left(\mathrm{CO}_{2}\right)$ in the surrounding air is increased to $30 \%(\mathrm{v} / \mathrm{v})$. Secondly, growth of the uridine mutant in $30 \% \mathrm{CO}_{2}$ in air is prevented by arginine; and the growth of the arginine mutant in $30 \% \mathrm{CO}_{2}$ in air is prevented by uridine. Some of these results have been presented elsewhere (Charles, 1962) but will be re-considered here in so far as they are relevant to a discussion of the relationship between uridine and arginine metabolism.

\section{METHODS}

Carbon dioxide and air mixtures. Carbon dioxide was generated by dilute hydrochloric acid and marble chips in a Kipps apparatus, any $\mathrm{HCl}$ being removed by bubbling the gas through sodium bicarbonate solution. Petri dish cultures were 
supplied with $\mathrm{CO}_{2}$ by placing them in $5 \mathrm{l}$. Pyrex vacuum desiccators, reducing the air pressure in the desiccator appropriately with an air pump, and then admitting $\mathrm{CO}_{2}$ until atmospheric pressure was restored. Restoration of atmospheric pressure was indicated by the swelling of a thin inflatable polythene bag interposed in the delivery tube from the Kipps apparatus.

Desiccators were charged at room temperature. When incubation was above room temperatures excess gas was released by quickly opening and closing the tap after the desiccators had stood in the incubator for a few minutes. When necessary, $\mathrm{CO}_{2}$-free air was obtained by placing about $100 \mathrm{ml} .50 \%(\mathrm{w} / \mathrm{v})$ potassium hydroxide solution in the appropriate desiccator. Tufts of glass wool were partially immersed in the potassium hydroxide solution to increase the area of $\mathrm{CO}_{2}$-absorbing surface. All desiccators were opened and refilled with fresh gas mixture every $24 \mathrm{hr}$. It is not claimed that the methods used for regulating $\mathrm{CO}_{2}$ pressure were exact; they were satisfactory in that they gave reproducible results.

Media. Solid media were used in all experiments. Liquid cultures were not used because special apparatus would have been required for mixing and bubbling the gas mixtures. Solid media also had advantages in that they enabled use of the auxanographic method for observing nutritional requirements, and permitted the observation of the behaviour of individual conidia.

The minimal culture medium was Fries $\mathbf{3}$ as modified by Ryan, Beadle \& Tatum (1943) solidified with $1 \%(\mathrm{w} / \mathrm{v})$ Difco Bacto agar. The sucrose concentration was $0.5 \%$, and $1 \%$ sorbose was added to induce colonial growth. The sorbose solution was autoclaved $\left(120^{\circ}, 20 \mathrm{~min}\right.$.) separately in concentrated solution and added to the rest of the medium immediately before it was poured into $9 \mathrm{~cm}$. Petri dishes.

Growth factor requirements, inhibitions and antagonisms were investigated by the auxanographic method (Pontecorvo, 1949). Unsterilized crystals of compounds were used in preliminary tests, but all important observations were confirmed with sterile solutions of known concentration. All solutions were adjusted to $\mathrm{pH} \mathbf{5} \cdot \mathbf{4}$.

Solid media in Petri dishes were inoculated by spreading a suspension of conidia over the dried surface of the medium or by suspending conidia in the molten medium just before it was distributed into Petri dishes. The second method was preferred because it gave a more uniform distribution of conidia, but it was usually unsuitable for experiments in which the effect of the gas-phase on growth was being examined.

In experiments with yeasts certain Petri-dish cultures were found to be more easily deprived of $\mathrm{CO}_{2}$ than others when potassium hydroxide was present in the desiccator. This was because the halves of some Petri dishes fitted together tightly enough to impede diffusion of gases. An attempt was made to overcome this difficulty by using glass rods $10 \mathrm{~cm}$. long or S-shaped wire hooks to hold the halves of the dishes slightly apart at one edge. This method was unsatisfactory because the piles of Petri dishes were unstable and occupied more desiccator space. A better method found was to remove the outer halves (i.e. the lids) from the dishes and to incubate only the inner halves containing medium in the desiccators, with the exposed agar surfaces downwards. The first layer of three dishes rested on a wire shelf ( $\frac{3}{4}$ in. mesh) which covered the well of the desiccator. Successive layers of three dishes were stacked so that each dish rested equally on two of the dishes in the layer below. This arrangement permitted adequate diffusion of gases, and more dishes could be placed in each desiccator than by other stackings. Contamination 
of cultures did not increase when this method was used, and it was used whenever the effect of gas-phase composition on growth was being investigated. It was important to exclude air bubbles from the medium, particularly when attempting to free it from $\mathrm{CO}_{2}$. Some $\mathrm{CO}_{2}$-requiring mutants grew vigorously around air bubbles, even when incubated in a desiccator which contained potassium hydroxide. Presumably sufficient $\mathrm{CO}_{2}$ was then trapped in the air bubbles to enable growth to begin.

\section{RESULTS}

Fairley (1954) reported that a pyrimidine-requiring mutant of Neurospora (mutant 1298) grew when supplied with $\alpha$-aminobutyrate or threonine instead of the usual pyrimidines; Fairley's experiments were done in liquid media. Attempts by the present author to repeat Fairley's experiments on solid media were at first unsuccessful. When conidia of Neurospora mutant 1298 were plated on minimal medium $+\alpha$-aminobutyrate or threonine about one colony appeared for every $10^{4}$ viable conidia plated. Eventually it was found that when the conidia were incubated at $20^{\circ}$ they all gave colonies, whereas at $30^{\circ}$ no conidia grew. Thus the mutant was temperature sensitive when growing with $\alpha$-aminobutyrate or threonine. When the temperature of incubation was increased from $20^{\circ}$ to $30^{\circ}$, fewer and fewer conidia gave vigorous colonies. In contrast, the mutant was not temperature sensitive when supplied with pyrimidines, and then grew vigorously even at $30^{\circ}$. The most satisfactory explanation of this behaviour of mutant 1298 seemed to be that some kind of adaptive process was necessary to enable utilization of $\alpha$-aminobutyrate or threonine, and that the chances of the adaptation occurring decreased with increasing temperature. Mutation was excluded as an explanation because the rare colonies which appeared at high temperature gave cultures which were identical in all respects with mutant 1298.

Experiments with mutant 1298 were next done to determine whether any other environmental factors, apart from temperature, influenced the temperaturesensitive process. These experiments were unsuccessful until discovery of a reference by Haldane (1954) to the work of Borek \& Waelsch (1951) led to an examination of the effect of $\mathrm{CO}_{2}$ pressure on the mutant. Borek \& Waelsch showed that an instance of temperature sensitivity in certain lactobacilli was attributable to a requirement for $\mathrm{CO}_{2}$; as the incubation temperature was increased the $\mathrm{CO}_{2}$ concentration in the medium gradually decreased until at $30^{\circ}$ insufficient $\mathrm{CO}_{2}$ was dissolved for growth to occur. Borek \& Waelsch suggested that some instances of temperature sensitivity in mutants might be due 'to a need for $\mathrm{CO}_{2}$ in the medium'.

When incubated in a vacuum desiccator containing air $+15 \%(\mathrm{v} / \mathrm{v}) \mathrm{CO}_{2}$ conidia of Neurospora mutant 1298 grew vigorously on $\alpha$-aminobutyrate or threonine medium; germination of conidia and growth at $30^{\circ}$ were the same as at $20^{\circ}$. It thus appeared that Borek \& Waelsch's suggestion was applicable to mutant 1298, and that $\mathrm{CO}_{2}$ was required for growth on $\alpha$-aminobutyrate medium; with increasing temperature, less and less $\mathrm{CO}_{2}$ would be dissolved in the medium, and growth would become increasingly difficult. Possibly the rare conidia which grew at the higher temperatures did so because they were near air bubbles trapped in the agar. Once conidia have started to grow it is possible that they generate sufficient $\mathrm{CO}_{2}$ to enable growth to continue. The ability of rare conidia to grow in air at higher temperatures 
was not investigated experimentally : attention was directed towards other aspects of the $\mathrm{CO}_{2}$ effect.

It is suggested above that a certain concentration of $\mathrm{CO}_{2}$ is necessary to enable the mutant to respond to $\alpha$-aminobutyrate or threonine. If this be true, the mutant should not respond to $\alpha$-aminobutyrate when $\mathrm{CO}_{2}$ is completely removed from the air, even at $20^{\circ}$. Experiment confirmed this suggestion. Mutant 1298 did not respond to $\alpha$-aminobutyrate when incubated at $20^{\circ}$ in a $5 \mathrm{l}$. desiccator containing $100 \mathrm{ml} .50 \%(\mathrm{w} / \mathrm{v})$ potassium hydroxide. In contrast, the response of mutant 1298 to added uridine or other pyrimidines was not prevented by removing $\mathrm{CO}_{2}$ in this way. Wild-type conidia grew vigorously on minimal medium or on $\alpha$-aminobutyrate medium in the absence of $\mathrm{CO}_{2}$. The requirement for $\mathrm{CO}_{2}$ thus appeared to be characteristic of mutant 1298 when growing on $\alpha$-aminobutyrate or threonine medium. Further experiments revealed that mutant 1298 grew vigorously on minimal medium without supplement when the proportion of $\mathrm{CO}_{2}$ in the gas-phase was increased to $30 \%(\mathrm{v} / \mathrm{v})$. This mutant was not temperature-sensitive when incubated in an atmosphere containing $30 \%(\mathrm{v} / \mathrm{v}) \mathrm{CO}_{2}$. It was possible that the observed effects of $\mathrm{CO}_{2}$ resulted from an alteration of the $\mathrm{pH}$ value of the medium. However, growth of the mutant did not occur in air when the $\mathrm{pH}$ value of the minimal medium was adjusted in other ways. It therefore seems that $\mathrm{CO}_{2}$ did not act simply by disturbing the $\mathrm{pH}$ value of the medium.

To establish whether $30 \%(\mathrm{v} / \mathrm{v}) \mathrm{CO}_{2}$ in air completely overcomes the block in mutant 1298 it would be necessary to compare the growth rates of mutant and wildtype on minimal medium in air $+30 \% \mathrm{CO}_{2}$. This has not been done because of the technical difficulty of controlling the gas-phase in tubes of liquid media. However, it was clear from experiments with Petri-dish cultures that $30 \% \mathrm{CO}_{2}$ overcame most or all of the metabolic block in mutant 1298.

The above experiments show that Neurospora mutant 1298 may justifiably be referred to as a carbon dioxide-requiring mutant. Before the discovery of these $\mathrm{CO}_{2}$ effects it had been observed that yeast extract contained something which prevented growth of mutant 1298 on $\alpha$-aminobutyrate or threonine medium. These experiments were made at $20^{\circ}$ to obtain a vigorous growth response to $\alpha$-aminobutyrate in the control cultures. Arginine was found to reproduce the inhibitory effects of yeast extract. A low concentration of arginine (less than $1 \mathrm{mg}$./l.) caused complete inhibition of growth of mutant 1298 on $\alpha$-aminobutyrate or threonine media. The arginine inhibition was not competitive, in the sense that there was no concentration of arginine whose inhibitory effect could be overcome by increasing the concentration of $\alpha$-aminobutyrate or threonine. This conclusion was based on auxanographic experiments; when a spot of arginine solution was placed very near a spot of $\alpha$-aminobutyrate solution a zone of inhibition was obtained the radius of which was independent of $\alpha$-aminobutyrate concentration and dependent only on the arginine concentration.

Arginine did not prevent growth in the presence of uridine or other appropriate pyrimidines. Exposure of mutant 1298 to arginine therefore had the same result as depriving it of $\mathrm{CO}_{2}$, in that both treatments prevented growth on $\alpha$-aminobutyrate but did not prevent growth on pyrimidines. This suggested that arginine acted by preventing the mutant from responding to $\mathrm{CO}_{2}$. It was possible to test this hypothesis because the mutant responds to $30 \% \mathrm{CO}_{2}$ in the absence of $\alpha$-aminobutyrate 
Experiment confirmed that mutant 1298 was unable to grow in $30 \% \mathrm{CO}_{2}$ when arginine was present. The growth-promoting effects of $\alpha$-aminobutyrate and threonine were not investigated further.

The next question was: do any other Neurospora mutants respond to $\mathrm{CO}_{2}$ ? In principle $\mathrm{CO}_{2}$ might exert its effect on mutant 1298 in one of two ways. First, it might act as an essential precursor of some metabolite, such as uridine. Secondly, it might act in a physico-chemical way, perhaps by altering intracellular $\mathrm{pH}$ values or by combining with a protein and so altering its conformation. On either explanation it seemed that, if other $\mathrm{CO}_{2}$-requiring mutants occurred, some of them might be temperature sensitive when growing in air, in the same way that mutant 1298 growing on $\alpha$-aminobutyrate medium in air seemed to be temperature sensitive because of a relative deficiency of dissolved $\mathrm{CO}_{2}$ at the higher temperatures. Several temperature sensitive Neurospora mutants described by other workers were therefore tested, but none responded to $\mathrm{CO}_{2}$. A list of all mutants which have been tested and which did not respond to $30 \% \mathrm{CO}_{2}$ is shown in Table 1. For reasons to be discussed later arginine mutants were also tested for response to $30 \% \mathrm{CO}_{2}$. Of three mutants available, one (mutant 30300) grew vigorously on minimal medium in air $+30 \%(v / v) \mathrm{CO}_{2}$. This arginine mutant is 'leaky' in the sense that it grows slowly on minimal medium in ordinary air in the absence of arginine. When growing slowly on minimal medium in air, the hyphae are characteristically more submerged in the agar than would be the hyphae of wild-type Neurospora. The submerged growth of the hyphae of mutant 30300, and the knowledge that this mutant responded to $\mathrm{CO}_{2}$, suggested that the 'leaky' growth on minimal medium might be a response to the $\mathrm{CO}_{2}$ present (about $0.03 \%, \mathrm{v} / \mathrm{v}$ ) in ordinary air and medium. This suggestion was confirmed by finding that mutant 30300 did not grow on minimal medium when the Petri dishes of medium were incubated in a desiccator containing KOH. Mutant 30300 did not respond to $\alpha$-aminobutyrate or to threonine, under any conditions.

Since the response of the uridine mutant 1298 to $\mathrm{CO}_{2}$ was specifically prevented by arginine, a search was made to see whether the arginine mutant $\mathbf{3 0 3 0 0}$ was similarly inhibited by any common substance. Of many substances tested, uridine, cytidine, uracil, lysine and canavanine prevented growth of mutant 30300 on minimal medium in air $+30 \% \mathrm{CO}_{2}$. The three pyrimidines were not inhibitory when arginine was present; the inhibitions were annulled by arginine in a non-competitive manner. In contrast, the inhibitions by canavanine and lysine were competitively annulled by arginine. The pyrimidines therefore appear to inhibit specifically the response to $\mathrm{CO}_{2}$, whereas lysine and canavanine may be regarded as inhibiting a different stage, or more than one stage, of arginine metabolism. All these inhibitors prevented the 'leaky' growth of arginine mutant 30300 on minimal medium in air.

Uridine and cytidine were completely inhibitory at $1 \mathrm{mg} . / \mathrm{l}$; a higher concentration of uracil was required for complete inhibition. Other pyrimidines were ineffective or gave weak inhibitions at high concentrations. Those pyrimidines, such as cytosine and thymine, which did not support growth of mutant 1298 also did not inhibit the response of mutant 30300 to $\mathrm{CO}_{2}$.

Uridine, cytidine and uracil prevented all response to $\mathrm{CO}_{2}$ by mutant 30300 but they did not prevent growth stimulation by citrulline or arginine. Auxanographic experiments showed that there was no concentration of uridine which prevented 


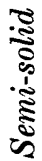

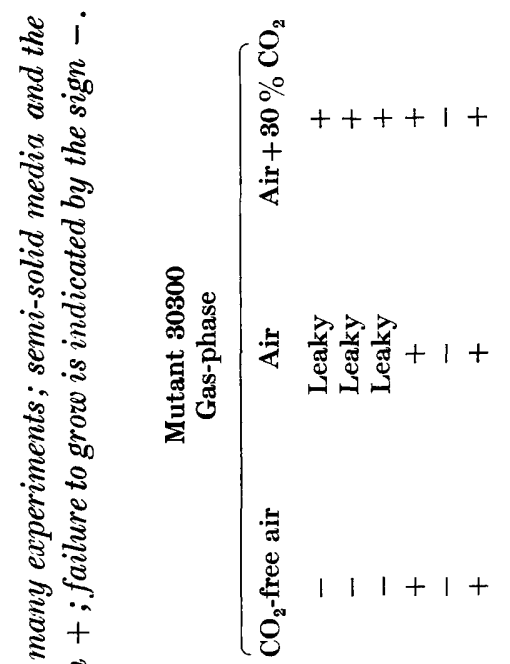

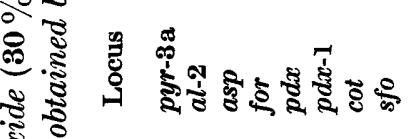

ปิ

is

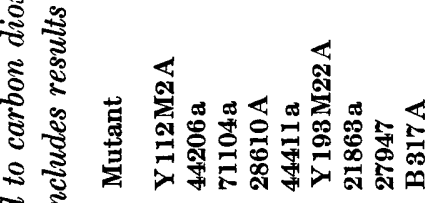

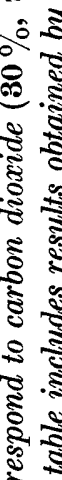

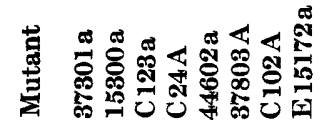

हิ

$\frac{5}{5}$

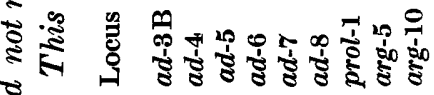

宽

)

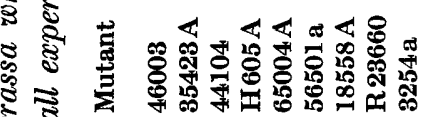

ล. .

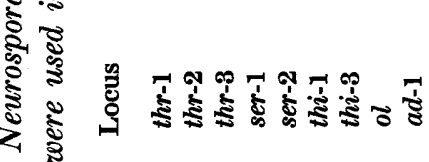

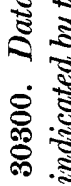

골

离

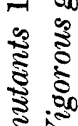

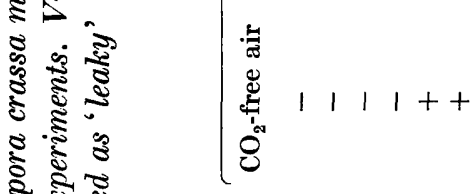

ถ⿻ำ

ฐँ๊

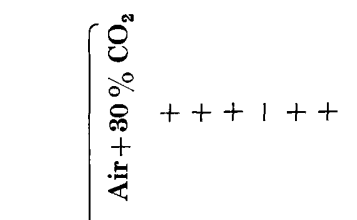

8

胥

20.

年

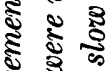

.

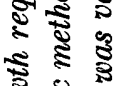

कू

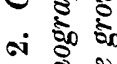

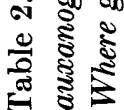

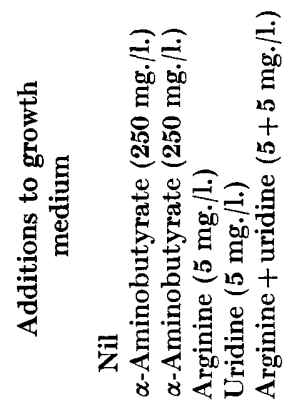

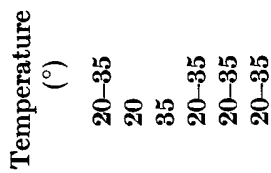


the growth response of mutant $\mathbf{3 0 3 0 0}$ to citrulline or arginine. These experiments were made in $\mathrm{C}_{2}$-free atmosphere; in ordinary air growth in presence of arginine was slightly inhibited by the pyrimidines. This slight inhibition is probably an inhibition of that part of the growth which is attributable to the $\mathrm{CO}_{2}$ of ordinary air.

The effects of $\mathrm{CO}_{2}$, arginine and uridine on mutants 1298 and 30300 are summarized in Table 2.

\section{DISCUSSION}

The experiments described above show that a Neurospora pyrimidine mutant (1298) and an arginine mutant (30300), unable to grow on a minimal medium alone, grew vigorously when the cultures were incubated in air $+30 \%(\mathrm{v} / \mathrm{v}) \mathrm{CO}_{2}$. The pyrimidine mutant 1298 did not grow on minimal medium in $30 \% \mathrm{CO}_{2}$ when arginine (1 mg./1.) was added; but when uridine was added mutant 1298 grew vigorously, whether or not $\mathrm{CO}_{2}$ was present in the air, and arginine was not inhibitory at any concentration. Conversely, arginine mutant $\mathbf{3 0 3 0 0}$ did not grow on minimal medium + uridine in $30 \% \mathrm{CO}_{2}$. With minimal medium + arginine, mutant 30300 grew vigorously, whether or not $\mathrm{CO}_{2}$ was present, and uridine was not inhibitory. The two mutants therefore exhibit an interesting symmetrical relationship. A similar relationship between certain pyrimidine and arginine mutants of Neurospora has also been shown through the study of suppressors of these mutations (Reissig, 1960; Davis, 1962).

The nature of the metabolic lesions in the mutants is not known. Davis (1960) studied Neurospora mutants allelic with mutant 1298 and found that the enzymes of the pyrimidine-synthesis pathway were unimpaired; Davis did not examine the enzymes which produce carbamoyl phosphate (see below). The first question is: how can an increase in the partial pressure of $\mathrm{CO}_{2}$ enable mutants 1298 and 30300 to grow? $\mathrm{CO}_{2}$ might act in two ways; it might act as a precursor of uridine or arginine, or it might act, for example, by altering intracellular $\mathrm{pH}$ value or by combining with a protein and altering its conformation. Of these two alternatives, only the 'precursor' hypothesis led to profitable experiments.

In developing the 'precursor' hypothesis, the obvious biochemical process through which $\mathrm{CO}_{2}$ might act is in the synthesis of carbamoyl phosphate, for which $\mathrm{CO}_{2}$ is known to be required; and carbamoyl phosphate in turn is a precursor of uridine and arginine (Jones, Spector \& Lipmann, 1955). Thus mutants 1298 and 30300 may have defects in the synthetic pathway to carbamoyl phosphate. The nutritional behaviour of arginine mutant $\mathbf{3 0 3 0 0}$ is consistent with the suggestion that it is blocked in the synthesis of carbamoyl phosphate, in that it grows when provided with citrulline but not when provided with ornithine. With regard to the pyrimidine mutant, as already pointed out, it is known that in Neurospora mutants similar to mutant 1298 the enzymes of the main pyrimidine pathway are all present (Davis, 1960) so that it is quite probable that one of the subsidiary processes which provide substances such as carbamoyl phosphate for the main pathway may be damaged.

The hypothesis of a defect in carbamoyl phosphate synthesis was originally developed to explain the behaviour of the pyrimidine mutant 1298, which was the first mutant found to respond to $\mathrm{CO}_{2}$ in place of pyrimidines. Since carbamoyl phosphate is involved in arginine metabolism as well as in pyrimidine metabolism, there seemed no reason why an arginine $\mathrm{CO}_{2}$-mutant should not also be found, if the 
explanation of the behaviour of the pyrimidine mutant were correct. This hypothesis therefore led to the testing of arginine mutants and the discovery that mutant 30300 grew on minimal medium in air $+30 \% \mathrm{CO}_{2}$.

If these mutants are defective in carbamoyl phosphate synthesis, there still remains the problem of how an increase in $\mathrm{CO}_{2}$ concentration enables these mutants to grow. The simplest explanation is that an enzyme which 'fixes' $\mathrm{CO}_{2}$ for carbamoyl phosphate synthesis has a decreased affinity for $\mathrm{CO}_{2}$, such that sufficient fixation of $\mathrm{CO}_{2}$ only occurs at a high $\mathrm{CO}_{2}$ concentration. This hypothesis is inadequate in two ways. First, the hypothesis leads one to expect that a single mutation which affects carbamoyl phosphate synthesis would lead to a mutant strain requiring arginine + a pyrimidine. It does not explain the occurrence of two different $\mathrm{CO}_{2}$ mutants, one of which responds alternatively to pyrimidine, the other alternatively to arginine. Secondly, the hypothesis does not explain why the growth of the pyrimidine mutant in air $+30 \% \mathrm{CO}_{2}$ is inhibited by arginine, nor why the growth of the arginine mutant in air $+30 \% \mathrm{CO}_{2}$ is inhibited by pyrimidines.

These considerations apparently require rejection of the hypothesis that both mutants are defective in carbamoyl phosphate synthesis because of an inadequate mechanism for fixing $\mathrm{CO}_{2}$. However, the fact that substances which the hypothesis predicts to be alternative growth factors turn out to be specific inhibitors seems unlikely to be mere coincidence. The hypothesis probably requires modification rather than rejection. Work by Stadtman, Cohen, Lebras \& de Robichon-Szulmajster (1961) on the biosynthesis of lysine, methionine and threonine by Escherichia coli provides a basis on which may be constructed a more satisfactory hypothesis. In $\boldsymbol{E}$. coli, lysine, methionine and threonine all have the same early precursors. Stadtman et al. (1961) obtained evidence that one of the common precursors, aspartyl phosphate, can be formed by three different enzymes (aspartokinases). One of these aspartokinases was specifically repressed and inhibited by lysine; the second aspartokinase was specifically inhibited by threonine; the third aspartokinase, for which the evidence was less complete, appeared to be inhibited by homoserine, a precursor of methionine. Different enzymes which catalyse the same chemical reaction are referred to as isozymes or isoenzymes (Markert \& Møller, 1959). By analogy, it is suggested that there may be two enzyme systems which synthesize carbamoyl phosphate in Neurospora, one system providing carbamoyl phosphate for uridine synthesis, and the other system providing carbamoyl phosphate for arginine synthesis. Such distinct pathways might be expected to be subject to regulation only by their own specific end-products. With certain further assumptions these Neurospora mutants can be understood if one assumes that the uridine mutant lacks an enzyme which produces carbamoyl phosphate for uridine synthesis, and that the arginine mutant lacks an enzyme which produces carbamoyl phosphate for arginine synthesis. Thus each of these mutants is considered to retain intact one mechanism for synthesizing carbamoyl phosphate, whereas the wild-type organism is considered to have both mechanisms (see Fig. 1). Spatial separation of the two enzyme systems appears to be necessary to explain why carbamoyl phosphate from one pathway is not freely available to the other defective pathway in these mutants.

Growth in air $+30 \% \mathrm{CO}_{2}$ would then be explained if the increased concentration of $\mathrm{CO}_{2}$ caused increased carbamoyl phosphate synthesis by the intact pathway, so 
that excess carbamoyl phosphate 'overflowed' and became available to the defective pathway. Thus the uridine mutant 1298 may be considered to respond to $\mathrm{CO}_{2}$ by utilizing excess carbamoyl phosphate synthesized by the arginine pathway. In the presence of exogenous arginine the latter pathway might be shut again by repression of enzyme formation or by feed-back inhibition, so that carbamoyl phosphate would no longer be synthesized from $\mathrm{CO}_{2}$. Thus the uridine mutant 1298 should be unable to respond to $\mathrm{CO}_{2}$ in the presence of arginine, although its ability to respond to pyrimidines should remain unchanged. Conversely, the arginine mutant 30300, when utilizing $\mathrm{CO}_{2}$, would be dependent upon the uridine pathway for formation

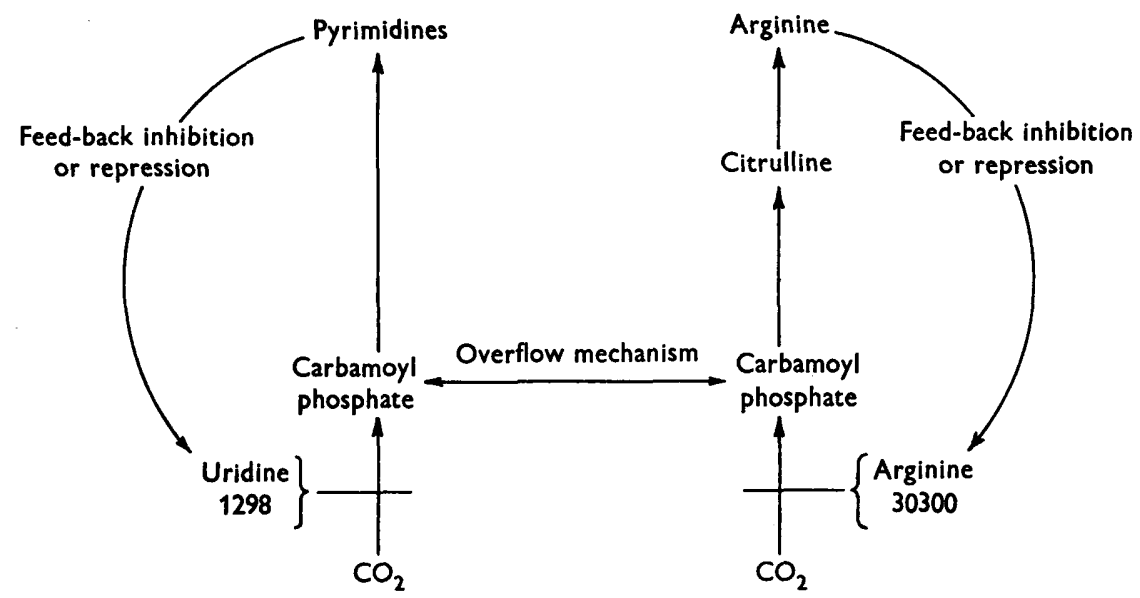

Fig. 1. To show the suggested mechanism for synthesizing carbamoyl phosphate by two separate pathways, one pathway subject to feed-back control by arginine, the other pathway subject to feed-back control by pyrimidines. The figure also shows the 'overflow' mechanism which may operate when the $\mathrm{CO}_{2}$ concentration is high.

of excess carbamoyl phosphate, and should therefore be susceptible to inhibition by uridine; response of the arginine mutant 30300 to arginine should not be prevented by uridine. Thus, the apparent cross-inhibitions of growth between uridine and arginine can be explained in terms of isozymes and orthodox feed-back processes.

The arginine mutant 30300 grows slowly in air $\left(0.03 \% \mathrm{CO}_{2}\right)$ but not in $\mathrm{CO}_{2}$-free air; presumably the $\mathrm{CO}_{2}$ concentration in ordinary air leads to some overflow of carbamoyl phosphate from the uridine-specific pathway. This suggestion is supported by the fact that this 'leaky' growth does not occur when uridine is present. The uridine mutant 1298 does not show 'leaky' growth, suggesting that there is no overflow of carbamoyl phosphate from the arginine pathway when this mutant is incubated in air.

Enzymological and genetical studies by Davis $(1960,1961)$ and Davis \& Woodward (1962) on 'suppressed' pyrimidine mutants support the hypothesis presented here. These workers studied a suppressor mutation which enables mutants similar to pyrimidine mutant 1298 to grow on minimal medium (Houlahan \& Mitchell, 1947, 1948; Mitchell \& Mitchell, 1952). Davis \& Woodward did not study the enzymes responsible for carbamoyl phosphate synthesis, but they found that the enzymes catalysing the subsequent steps of the pyrimidine pathway were not 
changed by the original mutations, regardless of whether the suppressor mutation was present or not. However, when the suppressor mutation was present the mutants showed a considerable decrease in ornithine transcarbamylase activity, having less than $2 \%$ of the enzymatic activity of the unsuppressed mutants and of wild-type cultures. One of the effects of the suppressor was thus to limit the amount of free arginine in the mycelium, without actually leading to an arginine deficiency. As Davis (1961) pointed out, growth of the pyrimidine-less mutants when arginine synthesis is diminished suggests that the unsuppressed mutants do not grow because of an internal inhibition by endogenously produced arginine. In terms of the hypothesis developed here, synthesis of carbamoyl phosphate for the arginine pathway would normally be regulated by an arginine feed-back mechanism such that sufficient carbamoyl phosphate is produced to meet only the needs of the arginine pathway. Diminution of the free arginine pool by the suppressor mutation might well permit synthesis of excess carbamoyl phosphate such that some becomes available to the uridine pathway, thereby enabling the pyrimidine mutant to grow. Significantly, the suppressed mutants are very susceptible to inhibition by exogenous arginine (Houlahan \& Mitchell, 1947). Davis (1962) suggested that there might be two sources of carbamoyl phosphate in Neurospora, one specific for uridine synthesis, the other specific for arginine synthesis.

Other observations which may be explicable in terms of mutations in isozymic systems are worth consideration. Miller \& Harrison (1950) described a yeast which was unusual in being strongly inhibited by uracil; this uracil inhibition was annulled non-competitively by arginine. This yeast thus behaved rather like the Neurospora arginine mutant 30300. It is possible that the yeast of Miller \& Harrison was deficient in a carbamoyl phosphate-synthesizing system which was specific for arginine synthesis and had therefore become completely dependent on a carbamoyl phosphate-synthesizing system normally specific for pyrimidine metabolism. That uracil was the most efficient inhibitor suggests that uracil might be a feed-back regulator of pyrimidine synthesis in this yeast; it would be necessary to assume that the regulatory system in this yeast is such that adequate carbamoyl phosphate normally overflows to supply the arginine pathway.

Doudney \& Wagner (1952) described a Neurospora mutant which grew well on minimal media but differed from the wild-type in being strongly inhibited by threonine, and the threonine inhibition was annulled non-competitively by methionine. Reference has already been made to the work of Stadtman et al. (1961) on the synthesis of threonine and methionine by Escherichia coli. By adapting the hypothesis developed above for the arginine and uridine $\mathrm{CO}_{2}$-mutants of Neurospora, the mutant of Doudney \& Wagner may be readily understood as having lost an isozyme from the early part of the methionine pathway. Since vigorous growth occurred on minimal medium, it would seem that adequate precursors overflowed from the intact threonine pathway, where the early steps are the same as in methionine synthesis. An external supply of threonine would be expected to prevent the production of these precursors, thereby explaining the inhibitory effect of threonine and its non-competitive annulment by methionine.

The situations discussed above suggest certain general principles applicable to the study of the genetics of mutations in isozymic systems. The simplest case may be considered, namely, the occurrence of two isozymic pathways within a cell, but 
the argument is applicable to multiple pathways such as those studied by Stadtman et al. First, if 'overflow' of identical precursors from one pathway to another does not occur, then mutation resulting in loss of an enzyme from one pathway should give a typical biochemical mutant which cannot grow on minimal medium. Since one isozyme would still be present, however, crude enzymological studies would not detect an enzyme deficiency in the mutant. Secondly, if restricted overflow takes place between the pathways, partially by-passing the block, then a slow-growing or 'leaky' mutant should result. Thirdly, if considerable overflow should occur, loss of one isozyme should give a mutant which grows vigorously on minimal medium. In both cases where overflow occurs the growth on minimal medium should be very sensitive to inhibition by the end-product of the intact pathway; this inhibition, in turn, should be overcome by the end-product of the damaged pathway, usually without competition.

At first sight it would seem that one cannot study the genetics of those isozymic systems where considerable overflow occurs, because the mutants should grow vigorously on minimal medium, and therefore would not be isolated by the usual screening techniques for obtaining mutants. However, sensitivity to inhibition by the appropriate end-product should offer a valuable method for increasing the efficiency of selection of 'leaky' mutants, and for detecting them once obtained. Stadtman et al. (1961) have already pointed out that the occurrence of 'leaky' mutants at a locus may offer useful evidence that an isozymic system is involved. Susceptibility of such 'leaky' mutants to inhibition by an appropriate end-product should be valuable supporting evidence for the presence of an isozymic system, especially when the inhibition is non-competitively annulled by a possible endproduct. Not many enzyme systems have been studied genetically. In some cases this is probably because the appropriate mutants are not isolated by the usual isolation procedures. The presence of isozymic systems, and consequent 'leaky' or 'very leaky' mutants, may account for the failure to isolate some of these mutants. This is probably true, for instance, of the early stages of lysine, threonine and methionine synthesis in micro-organisms.

Another situation which may be explicable in terms of isozymes is the occurrence of more gene loci than would be expected from the number of enzymes which control a given metabolic pathway. For example, there seem to be more genetic loci than known enzymes concerned with arginine synthesis in Neurospora. Since the arginine pathway may have other functions apart from the synthesis of arginine for proteins, it is possible that two or more isozymic pathways for synthesizing arginine occur in Neurospora. If this be the case, there must be little overflow between the pathways, otherwise the mutants would be too 'leaky' to be detected. It is interesting in this respect that arginine mutants seem to be uncommon in Salmonella. Perhaps in Salmonella two or more pathways for arginine synthesis occur, with considerable overflow of intermediate products between the two pathways, so that mutants deficient in an enzyme from one pathway are usually very leaky, and therefore not distinguishable from the wild-type organism.

Neurospora cultures were kindly provided by Professor D. G. Catcheside, F.R.S., and by Mr W. N. Ogata of the Fungal Genetics Stock Center (Dartmouth College, Hanover, New Hampshire, U.S.A.). I am grateful to Professor B. C. J. G. Knight for criticizing the manuscript, and to Mr J. A. Broadbent (working with a D.S.I.R. studentship) for some data in Table 1. 


\section{REFERENCES}

Borek, E. \& WAELSCH, H. (1951). The effect of temperature on the nutritional requirement of micro-organisms. J. biol. Chem. 190, 191.

Charles, H. P. (1962). Response of Neurospora mutants to carbon dioxide. Nature, Lond. 195, 359.

Davis, R. H. (1960). An enzymatic difference among pyr-3 mutants of Neurospora crassa. Proc. nat. Acad. Sci., Wash. 46, 677.

Davis, R. H. (1961). Suppressor of pyrimidine-8 mutants of Neurospora and its relation to arginine synthesis. Science, 134, 470 .

Davis, R. H. (1962). Consequences of a suppressor gene effective with pyrimidine and proline mutants of Neurospora. Genetics, 47, 351.

Davis, R. H. \& Woodward, V. W. (1962). The relationship between gene suppression and aspartate transcarbamylase activity in pyr-3 mutants of Neurospora. Genetics, 47, 1075.

DoudNey, C. O. \& WAGNER, R. P. (1952). Threonine inhibition in a strain of Neurospora. Proc. nat. Acad. Sci., Wash. 38, 196.

FAIRLEY, J. L. (1954). The growth-promoting effect of certain amino acids for pyrimidineless Neurospora mutants. J. biol. Chem. 210, 347.

Haldane, J. B. S. (1954). The Biochemistry of Genetics. London: George Allen and Unwin.

Houlahan, M. B. \& Mrtchell, H. K. (1947). A suppressor in Neurospora and its use as evidence for allelism. Proc. nat. Acad. Sci., Wash. 33, 223.

Houlahan, M. B. \& Mrtchell, H. K. (1948). Evidence for an interrelation in the metabolism of lysine, arginine and pyrimidines in Neurospora. Proc. nat. Acad. Sci., Wash. 34, 465 .

Jones, M. E., Spector, L. \& Lipmann, F. (1955). Carbamyl phosphate, the carbamyl donor in enzymatic citrulline synthesis. J. Amer. Chem. Soc. 77, 819.

MARKERT, C. L. \& MøLuER, F. (1959). Multiple forms of enzymes: tissue, ontogenetic and species specific patterns. Proc. nat. Acad. Sci., Wash. 45, 753.

Miller, E. J. \& HARrison, J. S. (1950). Growth inhibition of a yeast by uracil, and its reversal by arginine. Nature, Lond. 166, 1035.

Mitchell, M. B. \& Mrtchell, H. K. (1952). Observations on the behaviour of suppressors in Neurospora. Proc. nat. Acad. Sci., Wash. 38, 205.

Ponteconvo, G. (1949). Auxanographic techniques in biochemical genetics. J. gen. Microbiol. 3, 122.

Reissig, J. L. (1960). Forward and back mutation in the pyr-3 region of Neurospora. I. Mutations from arginine dependence to prototrophy. Genet. Res. 1, 356.

Ryan, F. J., Beadle, G. W. \& TAtum, E. L. (1943). The tube method of measuring the growth rate of Neurospora. Amer. J. Bot. 30, 784.

Stadtman, E. R., Cohen, G. N., Lebras, G. \& de Robichon-Szulmajster, H. (1961). Feed-back inhibition and repression of aspartokinase activity in Escherichia coli and Saccharomyces cerevisiae. J. biol. Chem. 236, 2033. 